\title{
An injection olfactometer for humans and a new method for the measurement of the shape of the olfactory pulse
}

\author{
RENÉ A. DE WIJK \\ University of Utrecht, Utrecht, The Netherlands \\ WIM VAESSEN and JOHANNES HEIDEMA \\ Unilever Research Laboratory, Vlaardingen, The Netherlands \\ and \\ E. P. KÖSTER \\ University of Utrecht, Utrecht, The Netherlands
}

\begin{abstract}
An injection olfactometer for human psychophysical experiments is described. The olfactometer is capable of presenting, either mono- or birhinally, single odorants and binary mixtures. The pulse form of the olfactory stimulus at the exit of the olfactometer approaches a rectangular form, which makes the olfactometer well suited for olfactory reaction time measurements. Since stimulation periods are independent of the subject's inhalation, time-intensity measurements over prolonged periods of time are possible as well. In the second part of this paper, a new technique is described for the measurement of the stimulus pulse form at the exit of the olfactometer.
\end{abstract}

In olfactory research, olfactometers are used as a tool to present stimuli of different odorous qualities and welldefined concentrations to either human or animal subjects. Three stimulus variables are especially important for olfactory perception: stimulus concentration, stimulus flow rate, and stimulus duration. The predominant influence of these variables on olfactory perception has been demonstrated by a number of investigators (de Wijk, 1989; Kobal, 1981; Laing, 1982; Overbosch, de Wijk, de Jonge, \& Köster, 1989; Rehn, 1978). In most cases, the control of these variables is limited by the fact that the stimulus is presented to the subject by way of the subject's inhalation. Consequently, the stimulus duration is limited by the duration of an inhalation, and the flow rate is not constant. Additionally, individual differences occur in flow rates and inhalation durations. To avoid these stimulus control problems, some olfactometers inject the stimulus directly into the nose of the subjects. As a result, stimulus presentation is independent of the subject's breathing, and, consequently, duration and flow rate can be controlled. One of the first injection olfactometers was developed by Elsberg and Levy (1935). Other injection olfactometers were developed by Stuiver

This research was supported by NWO Grant 560-262-016. The authors thank Gerd Kobal and Tommy Hendrikx for their valuable olfactometric advice, and Bill Cain for his support during the preparation of this manuscript. W. V. is now at Essenor, Delft, The Netherlands. Correspondence should be addressed to R. A. de Wijk, Brown and Williamson Tobacco Co., 2600 Weaver Road, Macon, GA 31298-1799 (e-mail: renedewijk@aol.com).
(1958) and, more recently, by Benignus and Prah (1980) and Kobal (1981, 1985; Kobal \& Hummel, 1991).

In order to gather precise information on time-dependent aspects of olfactory sensations, an olfactometer that met the following criteria was required: (1) a method of stimulus presentation independent of the subject's inhalation; (2) fast delivery of the odorant into the nose (additionally, the olfactory pulse should approach a rectangular form); (3) availability of a wide range of stimulus durations; (4) availability of a wide range of stimulus concentrations to present stimuli at threshold and suprathreshold levels; (5) rapid shifting times between concentrations; (6) independent stimulus presentation of at least two different odorants into one or both nostrils. Additional criteria were to be met regarding aspects of the olfactometer influencing the subject's reactions and the subject's sensory condition during experimentation: (7) a constant flow through the subject's nose both in the case of stimulation and in the case of nonstimulation in order to ensure that the subject responds only to olfactory or trigeminal cues, and not to tactile or somatosensory cues; (8) humidity and temperature conditioning of the injection flow in order to avoid irritation during prolonged stimulus presentation. Finally, administrative aspects of experimentation defined two more criteria: (9) automatic presentation of series of two odorants with various concentrations and durations; (10) linkage of timing of stimulus presentation and response acquisition. Although other olfactometers met some of these criteria, none of these olfactometers provided a wide concentration range plus rapid shifting times between these concentrations (criteria 4 and 5). 


\section{THE OLFACTOMETER}

A schematic representation of the olfactometer is given in Figure 1. The olfactometer consists of two types of channels: air channels and nitrogen channels. The nitrogen channel consists of two odorant lines. Nitrogen was used for the odor lines because nitrogen is less reactive and cleaner than air. One odorant is vaporized in each odorant line, and the resulting odorant/nitrogen mixture can be diluted in several steps by means of three dilution units. During stimulation, the odorant/nitrogen flow is injected into one or both air flows (at A or B; see Figure 1), which lead to each of the subject's nostrils (L and R; see Figure 1). The configuration of both channels is discussed in more detail below.

\section{Air Channel}

The air is drawn in from outside the building. After being compressed and led through oil and carbon filters, the air is divided into two equal flows, each leading to one of the subject's nostrils. The flow rates are measured by flow meters (Brooks rota meters) and set by needle valves $C$ and $D$ (Nupro stainless steel fine-metering valves) at $4,300 \mathrm{ml} / \mathrm{min}$ (Figure 1). To raise the relative humidity of the air to a constant value of $80 \%$, each of the flows is led through a gas-washing bottle containing warm water at a constant temperature of $33^{\circ} \mathrm{C}$. Furthermore, both air ducts are surrounded by a water mantle. The water in this mantle is kept at $40^{\circ} \mathrm{C}$ and is circulated by a pump. The hot water heats the air flowing through the ducts. A similar method has been used by Kobal (1981, 1985; Kobal \& Hummel, 1991). At L and R (Figure 1), each air duct ends in a duct with a smaller diameter ( $1 / 8$ in. instead of $1 / 4$ in.). The smaller ducts end in replaceable Teflon nose pieces. During stimulation, the nose pieces are placed in each of the subject's nostrils. The humidified air at the nose pieces is at body temperature $\left(37^{\circ} \mathrm{C}\right)$, which is experienced as comfortable by the subjects.

\section{Nitrogen Channel}

This channel consists of two odor lines, each for the dilution of one odorant. Since these two odor lines are identical, only one will be described here and shown in Figure 1. Coming from an expansion vessel (custom-made from aluminum with a volume of $2 \mathrm{~L}$ ) that serves to re-

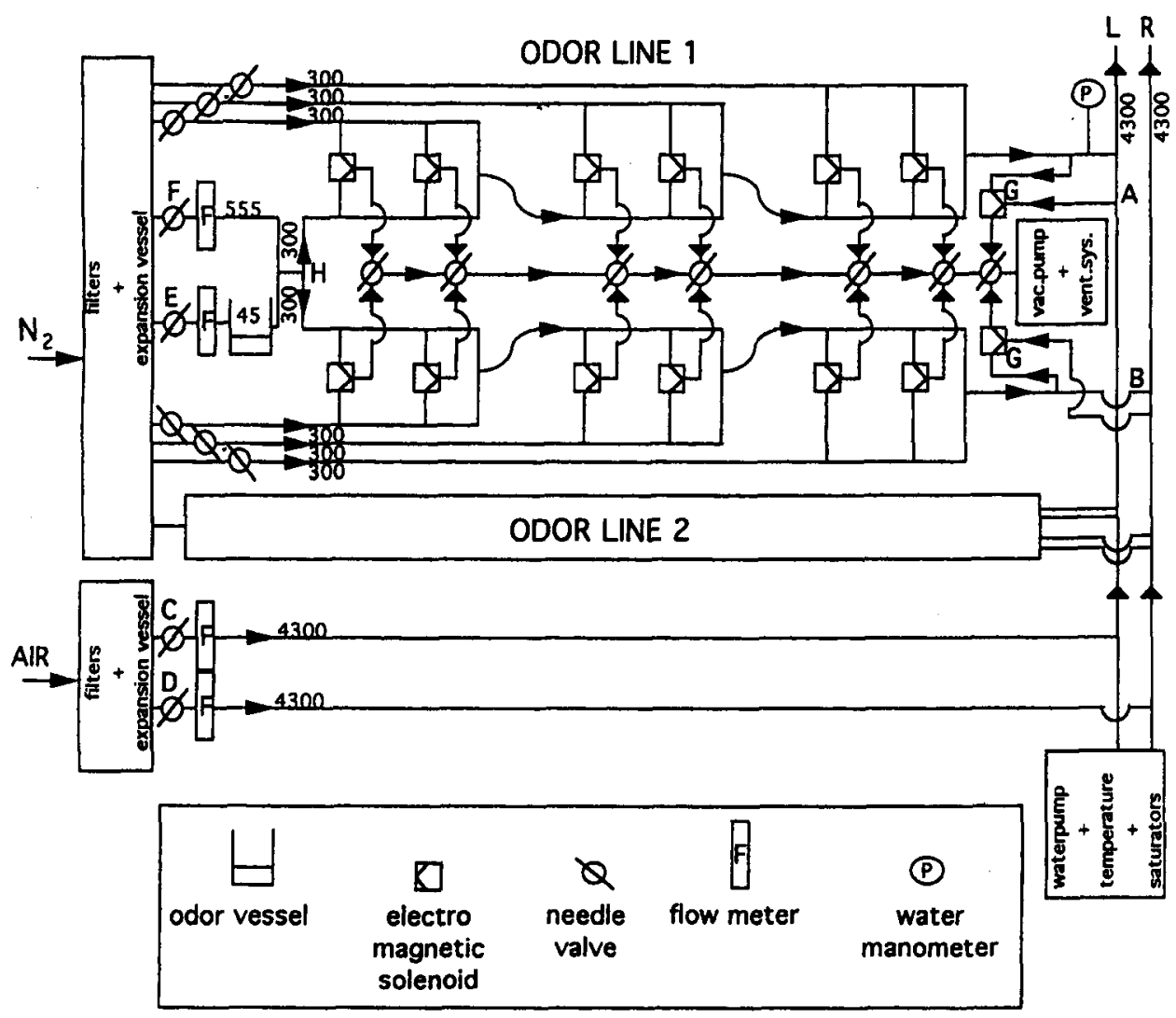

Figure 1. Injection olfactometer for the presentation of two odorants. The olfactometer consists of two channels: the air channel, which delivers $4,300 \mathrm{ml} / \mathrm{min}$ (controlled by needle valves $C$ and $D$ ) of filtered, warmed, and humidified air to each of the subject's nostrils ( $L$ and $R$ ), and the nitrogen channel, where odorant flows are generated (odor lines 1 and 2; odor line 1 is shown in detail). The amount of odorant flow is controlled by needle valves $E$ and $F$. Air and nitrogen channels are connected at $A$ and $B$. Injection of the odorant into the air flow takes place when the electromagnetic solenoids (G) are operated. The numbers refer to flow rates in milliliters per minute. 
duce fluctuations in the pressure of the incoming flow, thus creating a smooth outgoing flow, the flow of cleaned and filtered nitrogen is divided into two flows, which are controlled by needle valves $E$ and $F$ (see Figure 1) and measured by flow meters. Normally, the valves are set at a flow of 45 and $555 \mathrm{ml} / \mathrm{min}$, respectively. The smaller flow is led through a pair of odor vessels (two over-thesurface saturators, each with a length of $15 \mathrm{~cm}$ and a diameter of $1.5 \mathrm{~cm}$; see Dravnieks, 1975, for a more detailed description). The odor vessel is partly filled with an odorous liquid. The nitrogen flow carries the odorous vapor away with a maximal flow rate of $100 \mathrm{ml} / \mathrm{min}$, which is low enough to guarantee a saturated flow for almost all odorants (Dravnieks, 1975). The odor vessel is held at a fixed temperature of $15^{\circ} \mathrm{C}$ by means of a thermostatic bath. The odorous nitrogen flow is introduced into the larger flow, where the odorous flow is 13.3 -fold $(600 / 45)$ diluted (diluted to $7.5 \%$ of vapor saturation). In this way, an odorant flow of $600 \mathrm{ml} / \mathrm{min}$ is obtained.

When this odorant flow enters the odor line, it is split (at $\mathrm{H}$, see Figure 1) into two smaller flows of $300 \mathrm{ml} / \mathrm{min}$ each. Each flow passes three dilution units and is introduced into one or both of the air flows (at A and B, see Figure 1), which lead to the subject, in the case of stimulation.

A dilution unit is represented schematically in Figure 2 . The unit consists of an odorant flow $(300 \mathrm{ml} / \mathrm{min})$, an equally large nitrogen flow, two electromagnetic computer-controlled dilution solenoids $\mathrm{J}$ and $\mathrm{K}$ (Bürkert 3-way solenoids, $24 \mathrm{~V}$, response time $[\mathrm{RT}]=10 \mathrm{msec}$ ), and two needle exhaust valves ( 1 and 2) (Nupro stainless steel fine-metering valves). Depending on the setting of $\mathrm{J}$ and $\mathrm{K}$, some amount of flow is drawn away either from the odorant flow or from the nitrogen flow. Together, J and $\mathrm{K}$ draw away $300 \mathrm{ml} / \mathrm{min}$. Dilution takes place by drawing away some of the odorant flow and adding an equally large amount of odorless nitrogen to the remain-

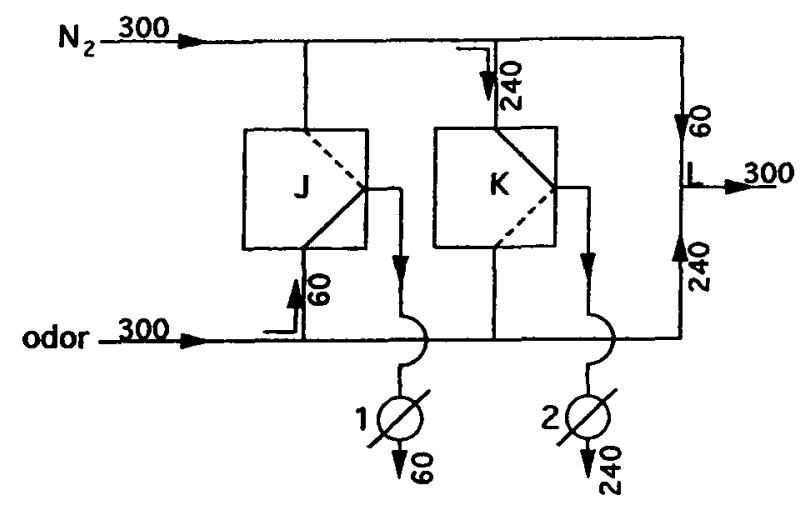

Figure 2. Example of a dilution unit consisting of an odorant flow $(300 \mathrm{ml} / \mathrm{min})$, a nitrogen flow $(300 \mathrm{ml} / \mathrm{min})$, two electromagnetic solenoids ( $J$ and $K$ ), and two exhaust needle valves ( 1 and 2 ). Combined, 1 and 2 draw away $300 \mathrm{ml} / \mathrm{min}$ from the olfactometer. In this example, $60 \mathrm{ml} / \mathrm{min}$ is drawn away from the odorant flow, and $240 \mathrm{ml} / \mathrm{min}$ is drawn away from the nitrogen flow. Consequently, the odorant flow is diluted by a factor of 1.2 at $\mathrm{L}$. Operating $\mathrm{J}$ and $\mathrm{K}$ results in a five times diluted odorant flow at $L$. ing odor flow. The result is a flow (at $\mathrm{L}$ ) with the same flow rate as the undiluted odorant flow $(300 \mathrm{ml} / \mathrm{min})$, but with a lower concentration of odor.

The dilution mechanism is described in more detail in the following example. The flows drawn away from the odorant and/or nitrogen flow are adjusted by means of exhaust needle valves 1 and 2 in such a way that 1 draws away approximately $1 / 5$ th $(=60 \mathrm{ml} / \mathrm{min})$ of a flow and 2 draws away the remaining $4 / 5$ th of that flow $(=240 \mathrm{ml} / \mathrm{min})$. Combined, 1 and 2 draw away the full amount of one flow (either odorant or nitrogen). An undiluted odorant flow at $\mathrm{L}$ is obtained if 1 and 2 draw away the full amount of nitrogen. Alternatively, the solenoids $\mathrm{J}$ and $\mathrm{K}$ can be operated in such a way that $1 / 5$ th of the odorant flow and $4 / 5$ th of the nitrogen flow are drawn away (this situation is depicted in Figure 2). Thus, $4 / 5$ th of the odorant flow is added to $1 / 5$ th of the nitrogen flow. This results in a 1.2-fold (or to $80 \%$ of its original concentration) diluted odorant at L. Finally, $J$ and $K$ can be operated in such a way that $4 / 5$ th of the odorant flow and $1 / 5$ th of the nitrogen flow is drawn away. This results in a 5 -fold (or to $20 \%$ of its original concentration) diluted odorant at $\mathrm{L}$.

Three dilution units, all with different dilution ratios, are placed serially. The dilution ratios are approximately $1 / 3$ and $2 / 3,1 / 4$ and $3 / 4$, and $1 / 5$ and $4 / 5$, respectively. From all possible combinations of the various dilution ratios, only 21 combinations were used. These combinations were chosen because they resulted in concentrations that were well spaced over the full concentration range.

Final dilution of the odorant takes place when the odorant flow is injected into the larger air flow (at $A$ and $B$; see Figure 1). This dilution factor is constant (4300/ $300=14.3$ ). Odorant dilution varies in 21 steps between $191(13.3 \times 14.3)$ and 11,460 -fold $(191 \times 60)$ (or between $0.0087 \%$ and $0.52 \%$ of vapor saturation) by means of the electromagnetic solenoids. Each additional dilution step further dilutes the odorant with a factor of approximately 1.25 . Figure $3 \mathrm{~A}$ shows an ion chromatogram, with relative selected ion abundances measured by mass spectrometer, for 16 of the 21 dilution steps. Although the dilution range is broad enough to cover both threshold and suprathreshold concentrations for most odorants, it can be changed by changing the flow through the odor vessel (maximum $100 \mathrm{ml} / \mathrm{min}$ ) by adding extra bypasses between the odor vessel and rest of the olfactometer, by increasing the surface area of the odorant in the odor vessel by lengthening the saturators, or by changing the temperature of the thermostat bath.

Injection of the odorant flow into the air flow takes place when the electromagnetic injection solenoid $G$ (see Figure 1) is operated in such a way that, instead of the odorant flow, an equally large amount of air is drawn away from the air flow; that is, part of the air flow is replaced by the odorant flow. The flow rate, and consequently the pressure measured by water manometer $P$ (Figure 1), remains, therefore, always the same, independent of the position of the electromagnetic dilution or injection solenoids. A similar injection system was first used in an olfactometer constructed by Kobal (1981). In 

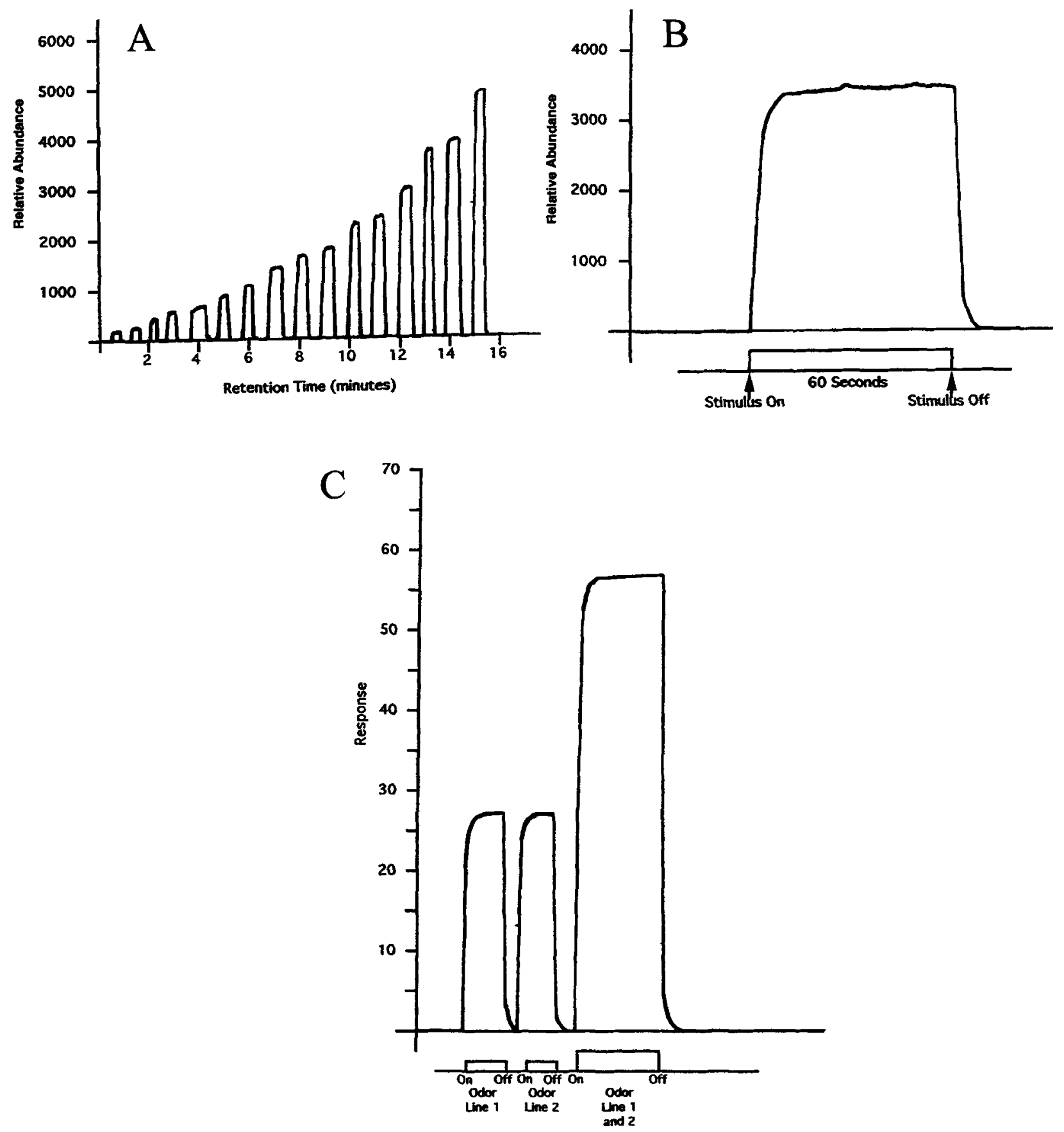

Figure 3. (A) Single real-time ion chromatogram $(M=2,200 \mathrm{~V}, \mathbf{M} / \mathbf{z}=81$, dwell $=20)$ of 16 dilution steps for $n$-hexane. The factor between steps equals approximately 1.25 . The concentration range for hexane is 8,853 to $354,116 \mathrm{ppb}$. (B) Single real-time ion chromatogram $(M=$ $2,200 \mathrm{~V}, \mathrm{M} / \mathrm{z}=68$, dwell $=20)$ of $d$-Limonene $(7,830 \mathrm{ppb}$ ) presented for $1 \mathrm{~min}$. (C) Single real-time concentration measurement with a Photo Ionization Detector of one concentration of $n$-hexane $(354,116 \mathrm{ppb})$ presented from odor line 1 , the same concentration of hexane from odor line 2, and a combination of the concentrations of hexane from odor lines 1 and 2.

the present olfactometer, solenoids are used not only for stimulus injection but also for stimulus dilution.

The concentration build-up of the stimulus at the nose pieces is fast due to the high linear speed of the flow through the last part of the olfactometer (between $A$ and $\mathrm{L}$, and $\mathrm{B}$ and R; see Figure 1), and due to the fact that the pressure in the olfactometer always remains the same.
The time delay between the moment that the injection solenoid is operated and the arrival of the first odor molecules at the nose pieces is calculated to be $170 \mathrm{msec}$. The subsequent time delay between the arrival of the first molecules at the nose pieces and the moment that the odor concentration has reached full strength is dependent on the odorant. The exact concentration build- 
up times for some odorants, measured at the nose pieces, are given in the final section of this paper.

\section{Materials}

The olfactometer is constructed in such a way that no cocks, needle valves, solenoids, capillaries, or other potential sources of contamination by odorants are placed in the ducts leading to the subject. Teflon and stainless steel are used as wall materials for the ducts. Only stainless steel was used for valves, fittings, and other parts. These materials are easy to clean in the case of contamination by odorants, and their adsorbing capacity is limited (Dravnieks, 1975).

\section{Calibration}

Calibration of the olfactometer involves the adjustment of the following: (1) the flow through each of the air ducts $(4,300 \mathrm{ml} / \mathrm{min})$; (2) the flow through each of the nitrogen ducts $(300 \mathrm{ml} / \mathrm{min}) ;(3)$ the flow through each of the odor vessels and bypasses; and (4) the flow drawn away from the olfactometer by means of the exhaust needle valves.

Adjustment of 1,2, and 3 is carried out by means of a combination of built-in needle valves and flow meters. First, the flow meters themselves were calibrated. Second, the function between flow rate and pressure increase, measured at $P$ (Figure 1), was established, which allows the use of pressure for flow calibration purposes. This eliminated the need for the installation of flow meters in every air or nitrogen duct.

Adjustment of each of the exhaust needle valves proved more difficult. The flow through each of these needle valves cannot be measured directly by means of (ball) flow meters, since odorous materials would stick to the parts of the flow meter, which would prevent the ball from floating freely through the flow meter. This would result in inaccurate measurements. Additionally, the large number of valves would require a large number of flow meters. For these reasons, another calibration method was developed, in which the water manometer (P; see Figure 1) was used. The amount of flow drawn away through each of the exhaust needle valves is a function of the differential pressure between the olfactometer and the underpressure in the ventilation system, caused by the vacuum pump. The differential pressure is controlled by the exhaust needle valve placed after each electromagnetic solenoid. If the underpressure is kept constant, the flow through each exhaust valve is a direct function of the pressure drop in the olfactometer caused by the flow drawn away. The pressure drop is measured by $\mathrm{P}$. Each exhaust valve is calibrated with only one flow $(300 \mathrm{ml} / \mathrm{min})$ entering the olfactometer, and all other exhaust needle valves are closed. To compensate for the change in underpressure when the olfactometer is fully functioning, a flow equal to the combined flow through all exhaust needle valves (except the one being calibrated) is injected directly into the ventilation system.

Afterward, when all exhaust needle valves had been calibrated separately, and the olfactometer was fully func- tioning, the result of the pressure calibration was checked by a Photo Ionization Detector (PID, HNU systems, Model PI-52) and by a Mass Spectrometer (HP MS 5970), which monitored one selected ion and used a gas chromatograph as inlet. The pressure calibration method proved to be satisfactory. Figure $3 \mathrm{~A}$ shows an ion chromatogram for the first 16 out of 21 dilution steps. The dilution steps are well distributed over the full concentration range.

A requirement for the use of time-intensity measurements is that the stimulus concentration delivered by the olfactometer must remain constant over prolonged periods of time. Figure 3B shows an ion chromatogram of one stimulus presented over a period of $1 \mathrm{~min}$. Indeed, the concentration remains constant.

Finally, for the presentation of binary mixtures it is essential that the concentration of one odorant at one nose piece not be affected by the simultaneous presentation of another odorant at the same nose piece. To test this, odor lines 1 and 2 were filled with the same odorant. The concentrations were measured both per odor line and for the two odor lines combined. Figure $3 \mathrm{C}$ shows that the concentration doubled when both stimuli were presented. Thus, the presentation of one odorant did not affect the simultaneous presentation of the other odorant.

The flow and concentration characteristics proved to be very constant over the first 2 years of use. Although the calibration was checked every day with the use of the water manometer, recalibration of flows was necessary only once every few months. After each experimental day, the olfactometer was flushed with clean air running through the odor lines. Thirty minutes before the start of an experiment, water was added to the gas-washing bottles, and the heating for the gas-washing bottles and water mantles surrounding the air ducts was switched on. Subsequently, all other flows and the vacuum pump were switched on.

\section{Stimulus Control and Response Acquisition}

The electromagnetic solenoids of the olfactometer are controlled by an Apple IIe computer. The commands are entered via the computer keyboard and executed by an interface. The electromagnetic solenoids are controlled either in a manual or in an automatic mode. The manual mode gives on-line control of the electromagnetic dilution and injection solenoids. In the automatic mode, an experimental file is created first. This file consists of codes of solenoids together with times at which the solenoid settings have to be executed (with an accuracy of $20 \mathrm{msec}$ ) during the automatic stimulus presentation (the software, written in Apple BASIC, and the interface were developed at the University of Utrecht).

Several response options are available: (1) For RT measurements, an Apple timer is started when the injection solenoid (G; see Figure 1) is operated. The timer is interrupted by a push button, pressed by the subject. Subsequently, RTs are registered by the computer with an accuracy of $\pm 25 \mathrm{msec}$. In view of the relatively long RTs in olfaction (ranging from approximately 500 to $4,000 \mathrm{msec}$ ), this accuracy was acceptable. (2) Perceived 
intensity measurements are carried out by means of a slide wire potentiometer with a length of $30 \mathrm{~cm}$. The perceived intensity is recorded either continuously over a prolonged period of time (time-intensity measurement with a maximum sample time of $240 \mathrm{sec}$, and a maximum sample rate of seven samples per second), or discretely at a predefined time period after the start of the stimulation. Perceived intensities are registered by a Kontron computer. Software, written in QBasic and developed at the Unilever Research Laboratory, has been used to analyze time-intensity measurements. The materials used, as well as the method of analysis, have been described by Overbosch, van den Enden, and Keur (1986).

\section{Subjects and Preliminary Results}

The olfactometer has been used extensively over a period of 2 years. Four subjects, between 23 and 28 years, were first practiced to use velopharyngeal closure during inhalation (see Kobal, 1981). With this method, the soft palate is pressed up against the pharynx to eliminate any flow between the nasal and oral cavities. Hence, activation of the gustatory system during stimulation of the olfactory system is eliminated. During practice, a fastreacting thermometer was fitted under the subject's nostrils. Inhalation or exhalation through the nose was revealed by a change in temperature. Within $15 \mathrm{~min}$, all subjects learned to breathe exclusively through the mouth. Subsequently, the subjects participated in a series of experiments that included measurements on detection thresholds and on the temporal processes of integration, adaptation, birhinal interaction, and mixture interaction. Detection thresholds obtained with the injection olfactometer were less sensitive than thresholds reported by others using methods in which odorants were inhaled by subjects. This difference in thresholds is probably related

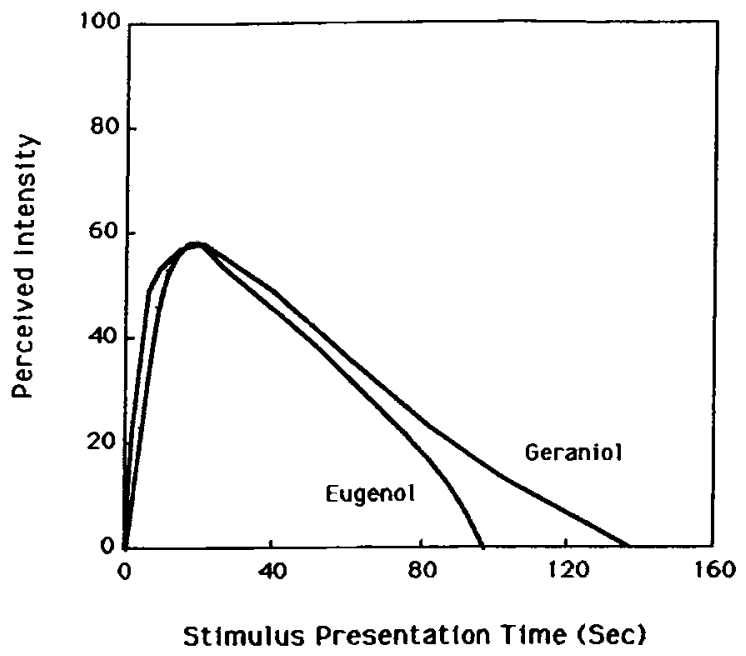

Figure 4. Examples of time intensity functions for 1 subject. The functions are the averaged results of 10 measurements per odorant. The concentrations of the geraniol and eugenol, 527 and $16 \mathrm{ppb}$, respectively, are equal to 10 times the individual threshold concentrations. Sample rate is $1 \mathrm{sample} / \mathrm{sec}$. to the relatively low stimulus flow rate of the olfactometer $(4.3 \mathrm{l} / \mathrm{min}$ vs. approximately $38 \mathrm{l} / \mathrm{min}$ for inhalations). Stuiver (1958) has demonstrated the negative relationship between detection threshold and stimulus flow rate. Olfactory RTs (ranging from 500 to $4,000 \mathrm{msec}$, depending on odorant and odorant concentration) and perceived intensities were recorded most often. Figure 4 shows an example of time-intensity measurements for one relatively slow-adapting odorant (geraniol) and one relatively fastadapting odorant (eugenol). Results have been reported elsewhere (de Wijk, 1989; Köster \& de Wijk, 1991; Overbosch et al., 1989).

\section{TENAX MEASUREMENTS}

Knowledge of the exact moment of presentation of the stimulus to the subject's nostrils, as well as knowledge of the exact concentration build-up (or pulse shape), is necessary for the accurate measurement of olfactory RTs, or for the recording of olfactory-evoked potentials (Evans, Kobal, Lorig, \& Prah, 1993). The actual shape of the olfactory pulse will always deviate from the ideal block form due to adsorption of the odorous molecules to the walls of the ducts of the olfactometer. The amount of adsorption is dependent on the odorant and on the construction of the olfactometer.

Several methods for the measurement of the pulse shape have been used by others. Examples of such methods are the measurement of the volume that leaves the olfactometer over a fixed period of time (Stuiver, 1958), and the use of a particle flow in which the particles are counted, or measured otherwise, when they leave the olfactometer (Kobal, 1981). The value of the results obtained with these methods is somewhat limited, because they do not take into account an important factor for the actual pulse shape, the odorant. Others have measured the pulse shape with the use of PIDs (Walker, Kurtz, Shore, Ogden, \& Reynolds, 1990). Our own experience with PIDs and with mass spectrometers has shown that such instruments are well suited for concentration measurements, but that their RTs are too long for accurate measurements of the concentration build-up (Figure 3A-3C).

A new method was developed by which the pulse shape is measured for experimental odorants. In the case of an instantaneous concentration build-up (see dashed line in Figure 5), the relationship between the stimulus duration (i.e., stimulus injection time), measured from the moment that the first molecules reach the nose pieces, and the cumulative amount of odor that leaves the olfactometer, is best described by a monotonic linear function. When the concentration build-up lags behind (see solid line in Figure 5) because of adsorption, this relationship is nonlinear for short stimulus injection times but becomes linear when the concentration build-up is completed that is, for longer stimulus injection times when the concentration that leaves the olfactometer has reached a steady state.

The cumulative amount of odor that leaves one nose piece of the olfactometer during various stimulus injection times is adsorbed by tubes (Supelco thermal desor- 


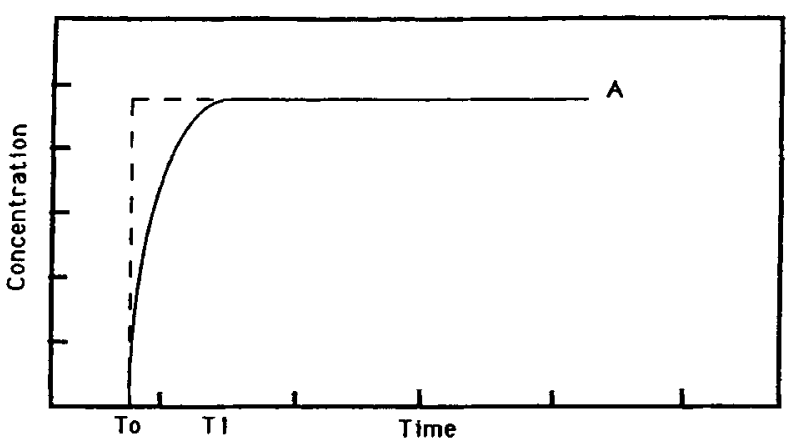

Figure 5. Theoretical function of stimulus concentration versus stimulus duration for a block-formed stimulus (dotted line) and for a deviation of the block form (solid line). The horizontal asymptote reflects Concentration $\mathrm{A}$.

bers, $11.5 \mathrm{~cm} \times 6 \mathrm{~mm}([\mathrm{OD}]) \times 4 \mathrm{~mm}$ [ID]) filled with Tenax-GC (Chrompack). Tenax-GC is a porous polymer based on 2,6,diphenyl-p-phenylene-oxide (PPS-771148 ); it is suitable for preconcentration of high boiling compounds, such as amines, alcohols, aldehydes, ketones, and aromatic compounds, on a gas chromatograph. Nonpolar organics can be retained as well (Pankow, Isabelle, \& Kristensen, 1982). A Tenax measurement is started when solenoid $G$ is operated and the full amount of odor/air flow that leaves the olfactometer is led through a pair of Tenax tubes. A large vacuum pump and a needle valve ensure that the amount of flow through the tubes is equal to the amount of flow that leaves the olfactometer. After various stimulus injection times, ranging from .1 to $30 \mathrm{sec}$ after $\mathrm{G}$ is operated, the flow through the Tenax vessels is terminated by a solenoid that redirects the flow through a bypass. Subsequently, the Tenax vessels are removed and the cumulative amount of odor in the vessels is measured with a gas chromatograph. The result of a series of Tenax measurements is a function of cumulative amount of odor versus stimulus injection time.

\section{Formal Description}

We chose to analyze the measured data with the use of a formal representation of the build-up process according to an exponential function, which results if we assume that the increase in loss of odor due to adsorption within the olfactometer is an exponentially decreasing function of time. Following that, the total amount of odor presented by the olfactometer, and adsorbed in the Tenax tubes $\left(\mathrm{Vol}_{\mathrm{ten}}\right)$, as a function of time $(t)$ for Concentration $\mathrm{A}$ of a given odorant is given by the following equation (see the Appendix for a derivation):

$$
\mathrm{Vol}_{\text {ten }}=\mathrm{A} *\left(t-1 / k_{1} *\left(1-\exp \left(-k_{1} * t\right)\right)\right)
$$

(for a curved pulse shape).

The ability of Tenax to adsorb a given volume of odorant is limited. The maximum volume retained by Tenax is determined by the breakthrough volume, denoted B. The volume of odor, as measured by a gas chromato- graph $\left(\mathrm{Vol}_{\text {chrom }}\right)$, relates, therefore, to the given volume $\left(\mathrm{Vol}_{\text {ten }}\right)$ as:

$$
\mathrm{Vol}_{\text {chrom }}=\mathrm{B} *\left(1-\exp \left(-k_{2} * \mathrm{Vol}_{\text {ten }}\right)\right)
$$

Data were collected for two odorants: $n$-hexane (presented in two concentrations) and 1,8 cineole. Tenax tubes were filled for periods ranging from 0.1 to $30 \mathrm{sec}$. Nonlinear regression analysis using PROC NLIN of SAS (SAS Institute, Inc., 1987) was used to fit the data of Table 1 to Equation 1. We weighted the observations with the reciprocal standard deviation for stabilizing the residual error. In this way we obtained estimated values for breakthrough volume (B) and the fitting parameters $k_{1}$ and $k_{2}$. Subsequently, the value of $T_{1}$ (Figure 6) was estimated as the point of intersection of the time axis and the tangent to the nonlinear regression curve. The resulting parameters and delay times are given in Table 2 .

It can be concluded that the build-up time of the concentration after the onset of the stimulation is less than $0.18 \mathrm{sec}$ for a high concentration of cineole. Apart from that, $0.17 \mathrm{sec}$ should be subtracted from reaction times, due to the time it takes for the odorant to travel from the injection solenoid to the nose piece.

\section{SUMMMARY AND CONCLUSIONS}

In summary, it can be concluded that the present olfactometer fulfills the criteria mentioned in the introduction: (1) Conditioning of the humidity and temperature of the

Table 1

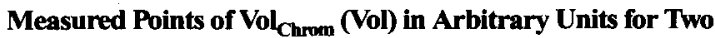
Concentrations of $n$-Hexane and One Concentration of 1,8 Cineole at Different Injection Times (IT)

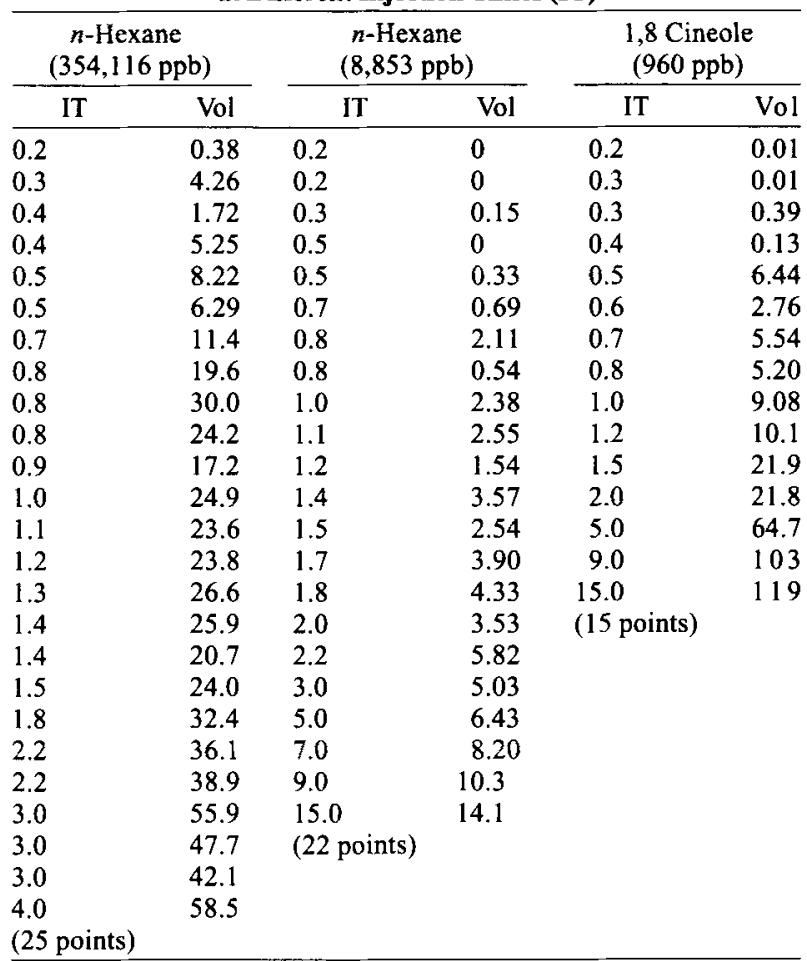



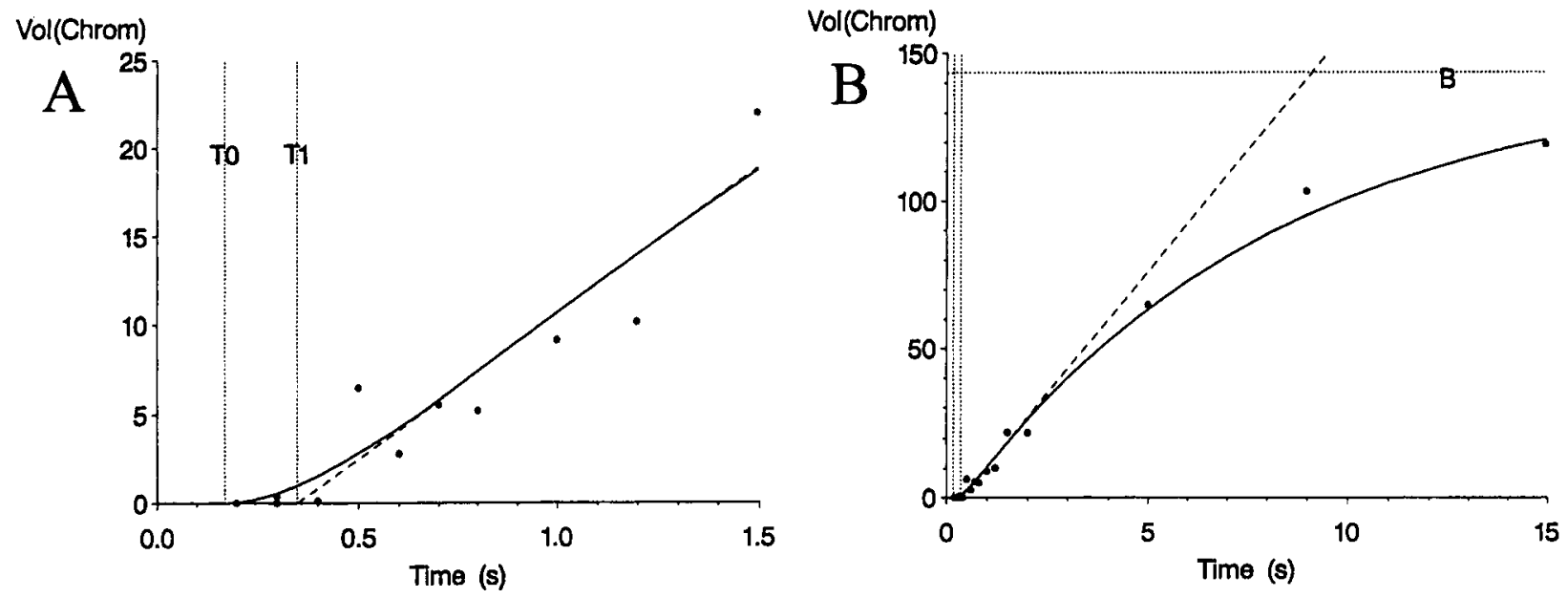

Figure 6. Amount of odorant $\left(\mathrm{Vol}_{\text {chrom }}\right.$ ) measured as a function of time. A: $T_{0}$ is the moment at which the injection solenoid is operated (i.e., the stimulation starts); $T_{1}$ is the inferred moment at which the odorant reaches the nose piece at Concentration $A$. B: The horizontal asymptote reflects the breakthrough volume (B; i.e., no more volume of odorant is adsorbed by the Tenax).

air flows is assured by leading the air flows through warmwater bottles and by surrounding the air ducts by a warmwater mantle. (2) A wide range of concentrations is achieved by leading the odorant flow through three dilution units, and the use of computer-controlled electromagnetic solenoids enables rapid shifts between concentrations. (3) The use of computer-controlled electromagnetic solenoids also enables the automatic presentation of stimulus series and the linking of stimulus presentation and response acquisition. (4) With regard to the aspects of the olfactometer that influence the subject's sensory conditions directly, it can be concluded that the mechanism of replacing odorant flows by equally large nitrogen flows ensures that flow rate, and, therefore, pressure at the olfactometer's exit, remains constant during periods of stimulation and nonstimulation. (5) The injected flow, and the special breathing technique of the subject, guarantees that stimulus presentation is independent of the subject's inhalation. (6) The injection mechanism enables the presentation of odorants over, in principle, unlimited periods of time. The mechanism is also suitable for the mono- or birhinal presentation of two odorants. (7) Finally, the olfactometer presents pulses that closely approach the rectangular shape, as was shown by the results of the Tenax measurements.

Table 2

Estimated Value of the Parameters for Two Concentrations of $n$-Hexane and One Concentration of 1,8 Cineole

\begin{tabular}{lcccc}
\hline & $\mathrm{B}$ & $k_{1}$ & $k_{2}$ & $T_{1}$ \\
\hline $\begin{array}{c}n \text {-Hexane } \\
(8853 \mathrm{ppb})\end{array}$ & 33.79 & $1.7 * 10^{7}$ & 0.06 & $<0.01$ \\
$\begin{array}{l}n \text {-Hexane } \\
(354,116 \mathrm{ppb})\end{array}$ & 58.94 & 26.3 & 0.58 & 0.03 \\
$\begin{array}{l}1,8 \text { Cineole } \\
(960 \mathrm{ppb})\end{array}$ & 143.40 & 4.4 & 0.13 & 0.18 \\
\hline
\end{tabular}

Note- $T_{1}$ is given in seconds.
Thus, the olfactometer is well suited for the investigation of time-dependent aspects of olfactory perception.

\section{REFERENCES}

Benignus, V. A., \& Prah, J. D. (1980). A computer-controlled vapordilution olfactometer. Behavior Research Methods \& Instrumentation, 12, 535-540.

DE WIJK, R. A. (1989). Temporal factors in human olfactory perception. Doctoral thesis, University of Utrecht, The Netherlands,

DravnieKs, A. (1975). Instrumental aspects of olfactometry. In D. G. Moulton, A. Turk, \& J. W. Johnston (Eds.), Methods in olfactory research (pp. 1-58). New York: Academic Press.

ElSBERG, C. A., \& LeVy, I. (1935). The sense of smell. A new and simple method of quantitative olfactometry. Bulletin of the Neurological Institute of New York, 4, 5-19.

Evans, W. J., Kobal, G., Lorig, T. S., \& Prah, J. D. (1993). Suggestions for collection and reporting of chemosensory (olfactory) eventrelated potentials. Chemical Senses, 18, 751-756.

KOBAL, G. (1981). Elektrophysiologische Untersuchungen des menschlichen Geruchsinns [Electrophysiological investigation of the human sense of smell]. New York: Thieme-Copythek.

KoBAL, G. (1985). Pain-related electrical potentials of the human nasal mucosa elicited by chemical stimulation. Pain, 22, 151-163.

Kobal, G., \& HummeL, T. (1991). Olfactory evoked potentials in humans. In T. V. Getchell, R. L. Doty, L. M. Bartoshuk, \& J. B. Snow, Jr. (Eds.), Smell and taste in health and disease (pp. 255-275). New York: Raven Press.

KösTER, E. P., \& DE WIJK, R. A. (1991). Olfactory adaptation. In D. G. Laing, R. L. Doty, \& W. Breipohl (Eds.), The human sense of smell (pp. 199-215). New York: Springer-Verlag.

LAING, D. G. (1982). Characterization of human behaviour during odour perception. Perception, 11, 221-230.

Overbosch, P., DE WiJK, R. A., DE JONGE, T. J. R., \& Köster, E. P. (1989). Temporal integration and reaction times in human smell. Physiology \& Behavior, 145, 615-626.

Overbosch, P., van dEN Enden, J. C., \& KeUR, B. M. (1986). An improved method for measuring perceived intensity/time relationships in human taste and smell. Chemical Senses, 11, 331-338.

PanKow, J. F., Isabelle, L. M., \& Kristensen, T. J. (1982). Effects of linear flow velocity and residence time on the retention of non-polar aqueous organic analytes by cartridges of Tenax-GC. Journal of Chromatography, 245, 31-43. 
REHN, T. (1978). Perceived odor intensity as a function of air flow through the nose. Sensory Processes, 2, 198-205.

SAS INSTITUTE, INC. (1987). SAS/STAT Guide for personal computers: Version 6. Cary, NC: Author.

Sturver, M. (1958). Biophysics of the sense of smell. Doctoral thesis, University of Groningen, The Netherlands.

Walker, J. C., Kurtz, D. B., Shore, F. M., Ogden, M. W., \& REYNOLDS, J. (1990). Apparatus for the automated measurement of the responses of humans to odorants. Chemical Senses, 15, 165 177

\section{APPENDIX}

When the concentration of a given pulse of odorant at the nose piece of the olfactometer equals $\mathrm{A}$, and the pulse shape is perfectly block-formed, the cumulative volume of odorant trapped in the Tenax tubes can be described easily. This volume of odorant gathered in the Tenax tubes over a certain period of time $(t)$ after the beginning of the stimulation would equal

$$
\mathrm{Vol}_{\text {ten }}=\mathrm{A} * t
$$

(for a block-formed pulse shape).

In the case of a deviation from the ideal block-formed shape, the Concentration A will not reach A immediately after the beginning of the stimulation. We chose to describe this build-up of concentration in time by an exponential function:

$$
\text { Conc }=\mathrm{A} *\left(1-\exp \left(-k_{1} * t\right)\right),
$$

which results if we assume that the increase in adsorption losses in the olfactometer is an exponentially decreasing function of time.

Depending on the value of the constant $k_{1}$, the concentration approaches the level of A more or less rapidly. In this case, the amount of volume retainable by the Tenax at the nose piece would equal

$$
\begin{aligned}
\mathrm{Vol}_{\text {ten }} & =(\mathrm{A} * t)-{ }_{0} \int t\left(\mathrm{~A} * \exp \left(-k_{1} * \mathrm{x}\right)\right) d x \\
\Leftrightarrow \quad \mathrm{Vol}_{\text {ten }} & =(\mathrm{A} * t)-\mathrm{A} / k_{1} *\left(-1-\exp \left(k_{1} * t\right)\right) \\
\Leftrightarrow \quad \mathrm{Vol}_{\text {ten }} & =\mathrm{A} *\left(t-1 / k_{1} *\left(1-\exp \left(-k_{1} * t\right)\right)\right)
\end{aligned}
$$

(for a curved formed pulse shape).

The ability of Tenax to adsorb odorants is, however, limited to a specific breakthrough volume (B). Again, we chose to describe the retained volume $\left(\mathrm{Vol}_{\text {ret }}\right)$ as a function of the input volume by an exponential curve:

$$
\mathrm{Vol}_{\text {ret }}=\mathrm{B} *\left(1-\exp \left(-k_{2} * \mathrm{Vol}_{\text {in }}\right)\right) \text {, }
$$

in which $k_{2}$ is a constant and $\mathrm{Vol}_{\text {in }}$ is the input volume. The volume of odorant as measured by the gas chromatograph ( $\mathrm{Vol}_{\text {chrom }}$ ) as a function of the volume available at the nose piece can be given accordingly:

$$
\mathrm{Vol}_{\text {chrom }}=\mathrm{B} *\left(1-\exp \left(-k_{2} * \mathrm{Vol}_{\text {ten }}\right)\right) \text {. }
$$

A simple substitution of $\mathrm{Vol}_{\text {ten }}$ would express the volume of odorant as measured by the chromatograph as a function of the Concentration A. Since A is given, and $\mathrm{Vol}_{\text {chrom }}$ is measured at different $t \mathrm{~s}$, values of the parameters $k_{1}$ and $k_{2}$ and the breakthrough Volume $B$ can be estimated by means of a nonlinear regression analysis. With the estimated values of these parameters, the value of $T_{1}$ (Figure 6) can be deduced. This value represents the estimated moment at which the full odor concentration reaches the nose piece of the olfactometer.

(Manuscript received February 3, 1992; revision accepted for publication June 19, 1995.) 